\title{
The effect of magnesium doped on microstructure of aluminum alloy under ultrasonic vibration
}

\author{
LI Chun ${ }^{1, a}$, LI Da ${ }^{2, b}$ and HUANG Wen Cheng ${ }^{3, c}$ \\ ${ }^{1-3}$ National Defence Key Discipline Laboratory of Light Alloy Processing Science and Technology, \\ Nanchang Hangkong University, Nanchang 330063, P. R. China \\ alichun7059@yahoo.com.cn, bdali1980@163.com, cwenchenghuang@163.com
}

Keywords: Ultrasonic vibration; Grain refinement; Microstructure

\begin{abstract}
Aluminum has great application in the machinery, transportation, aviation and military and other fields, because of its light weight, rich in resources, good comprehensive properties. Structure determines the properties, in order to obtain good properties, the key is to get the fine and uniform equiaxed grain. In order to obtain the high quality aluminum alloy of fine and uniform equiaxed grain, we must control the growth of grain in the casting process. This paper mainly discusses the effect of magnesium doped on microstructure and properties of aluminum alloy under ultrasonic vibration. Adding Mg of different content for $1 \mathrm{wt} \%$, 3wt $\%$, 5wt\%, 7wt $\%$, 9wt\%, $15 w t \%$ in pure aluminum, respectively, then solidifying under ultrasonic vibration. The effect of different solute content on the microstructure of aluminum alloy under ultrasonic vibration is studied. The result shows that aluminum alloy containing 9wt.\% magnesium can achieve the best effect of grain refinement under ultrasonic vibration.
\end{abstract}

\section{Introduction}

Grain refinement of $\mathrm{Al}$ and its alloys has been an important process in commercial production for over half a century because fine grains not only deliver enhanced casting soundness and mechanical properties of cast metals, but also facilitate the subsequent mechanical working and forming processing [1].

Dynamic nucleation or solidification, i.e., nucleation or solidification under dynamic conditions has been recognised and practiced for many years [2-3]. One of the most commonly used approaches to facilitate dynamic nucleation is that of ultrasonic vibration [3], which has been applied to the solidification of both metallic and non-metallic liquids. It has been reported that ultrasonic vibration can generate a high number density of fine ice crystals in the solidification of water within a short time [4]. In the area of the solidification of metallic materials, ultrasonic vibration can effectively grain-refine both aluminium and magnesium alloys [3,5]. Ultrasonic melt treatment (UST) is known to induce refining effect in aluminum alloys [6-11]. The basic principle is introduction of acoustic waves with a frequency higher than $17 \mathrm{kHz}$ into liquid metal. High frequency and high amplitude oscillations result in cavitation of the melt and also promote intense mixing through agitation. The reported mechanisms of refinement by UST range from affecting nucleation through local undercooling and wetting of substrates to fragmentation of forming crystals[6,10].

The grain size in pure ingots subjected to ultrasonic vibration during its solidification stage is still much larger compared to that in A356 alloy. Jian et al [12] tested grain refining in A356 alloy using an identical ultrasonic system. They poured A356 alloy melt at $634^{\circ} \mathrm{C}, 20^{\circ} \mathrm{C}$ above its liquidus, into a similar copper mold as used in this study, and obtained spherical grains. The size of the spherical grains was in the range of $40 \mu \mathrm{m}$ to $70 \mu \mathrm{m}$. This was almost one order of magnitude smaller than the size of equiaxed grains obtained in this study on pure aluminum ingots. The combination of using both grain refiners and ultrasonic vibration was then tested in the present study. This work reports our experimental observations on the effect of ultrasonic vibration and solute on the solidification structure of slowly cooled ingot samples of pure aluminum and Mg-doped aluminum alloys. The mechanisms by which ultrasonic vibration grain-refines aluminum 
are discussed on the basis of the cavitation study established on water and other molten alloys.

\section{Experimental Procedure}

Ingot samples ofpure aluminum (99.99\%), binary Al-Mg alloys containing 1\%, 3\%, 5\%, 7\%, and $9 \%$ of solute (all in wt.\%) were prepared with and without ultrasonic treatments in this study. A standard $20 \mathrm{kHz}$ high intensity commercial ultrasonic system was employed to apply the ultrasonic treatment. The ultrasonic system is comprised of an ultrasonic generator, an ultrasonic head, and a radiator. The horn and the radiator are made of a titanium alloy.

In each experiment, about $400 \mathrm{~g}$ of charge was melted in a boron nitride coated clay-graphite crucible in an electrical resistance furnace at $740{ }^{\circ} \mathrm{C}$. The coating was applied to prevent the pick-up of carbon by molten magnesium from the crucible.

Melting was conducted at certain temperature. After melting, the crucible containing the liquid metal was withdrawn from the furnace and placed in the air for ultrasonic treatment.

The ultrasonic system was switch on at the selected power before the vibration head was dipped into the liquid metal. The sonicating time was approximately 180 seconds. After sonicating, the vibration head was immediately withdrawn from the melt in the semisolid state and the ultrasonic source was switched off subsequently.

After cooling in the air the treated and untreated ingot samples were cut longitudinally through a central plane into two halves. One half was polished and etched using an hydrofluoric acid etching solution (10ml hydrofluoric acid $+90 \mathrm{ml}$ distilled water). The whole section of each polished half ingot sample was examined to study the effect of ultrasonic vibration on the resulting grain structures. The average grain size was measured according to the linear intercept method described in the ASTM standard E112-96.

\section{Results and discussion}

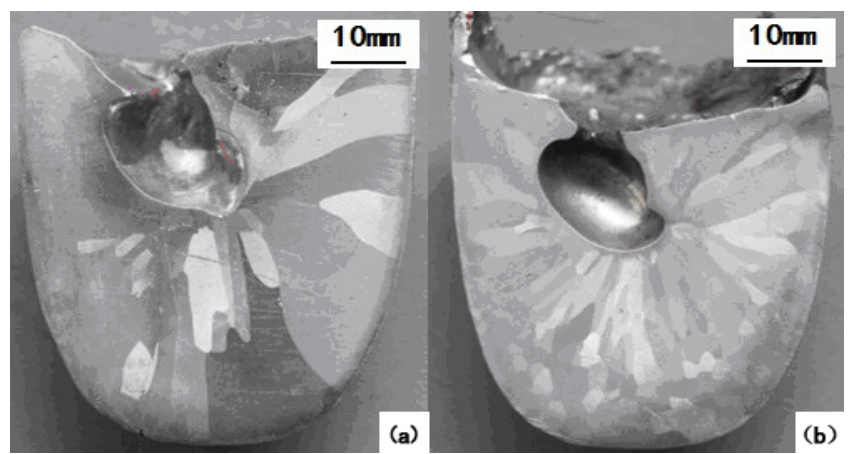

Fig. 1 Macrographs of the melted Al ingots: (a) without UV and (b) with UV treatment.

The initial macrostructures of the two kinds of as-cast pure $\mathrm{Al}$ are shown in Fig. 1: (a)without ultrasonic vibration and (b)with ultrasonic vibration treatment, respectively. Coarse dendrites of the Al phase were formed throughout the ingot as shown in Fig. 1(a). However, by applying UV to the melt, a relatively finer macrostructure was achieved as shown in Fig.1(b). Since the loss of acoustic energy increases the temperature gradient near the solidification front with the aid of mixing the melt with the strong acoustic flows, the formation of dendrite branches may be prevented. These grain refinement of the microstructures by the UV technique also have been observed in other alloys. 


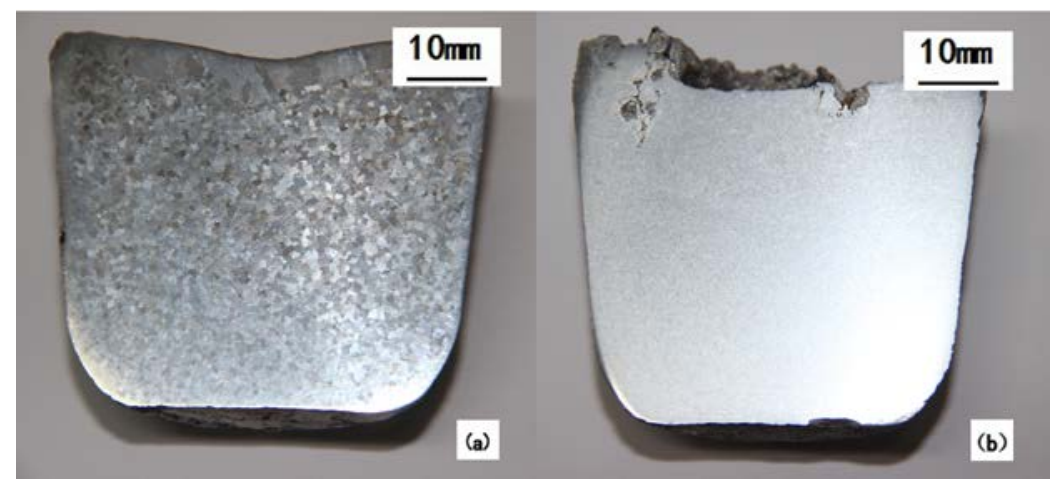

Fig. 2 Macrographs of the melted Al ingots under UV treatment: (a) with 7wt.\%Cu doping and (b) with 7wt.\%Mg doping.

We add the doping elements in the aluminum melts and then applying ultrasonic vibration, compared with pure aluminum, the grain refinement is more obvious which can be found in the doped Al alloys after the application of ultrasonic vibration, as shown in Fig. 2 and Fig. 3. Fig2 shows the macrographs of the melted aluminum melton under $\mathrm{UV}$ treatment with $7 \mathrm{wt} . \% \mathrm{Cu}, \mathrm{Mg}$ doping, respectively. This could explain that in the process of grain refinement, solute in the ultrasonic vibration conditions also play a big role, but its mechanism is still need further study.
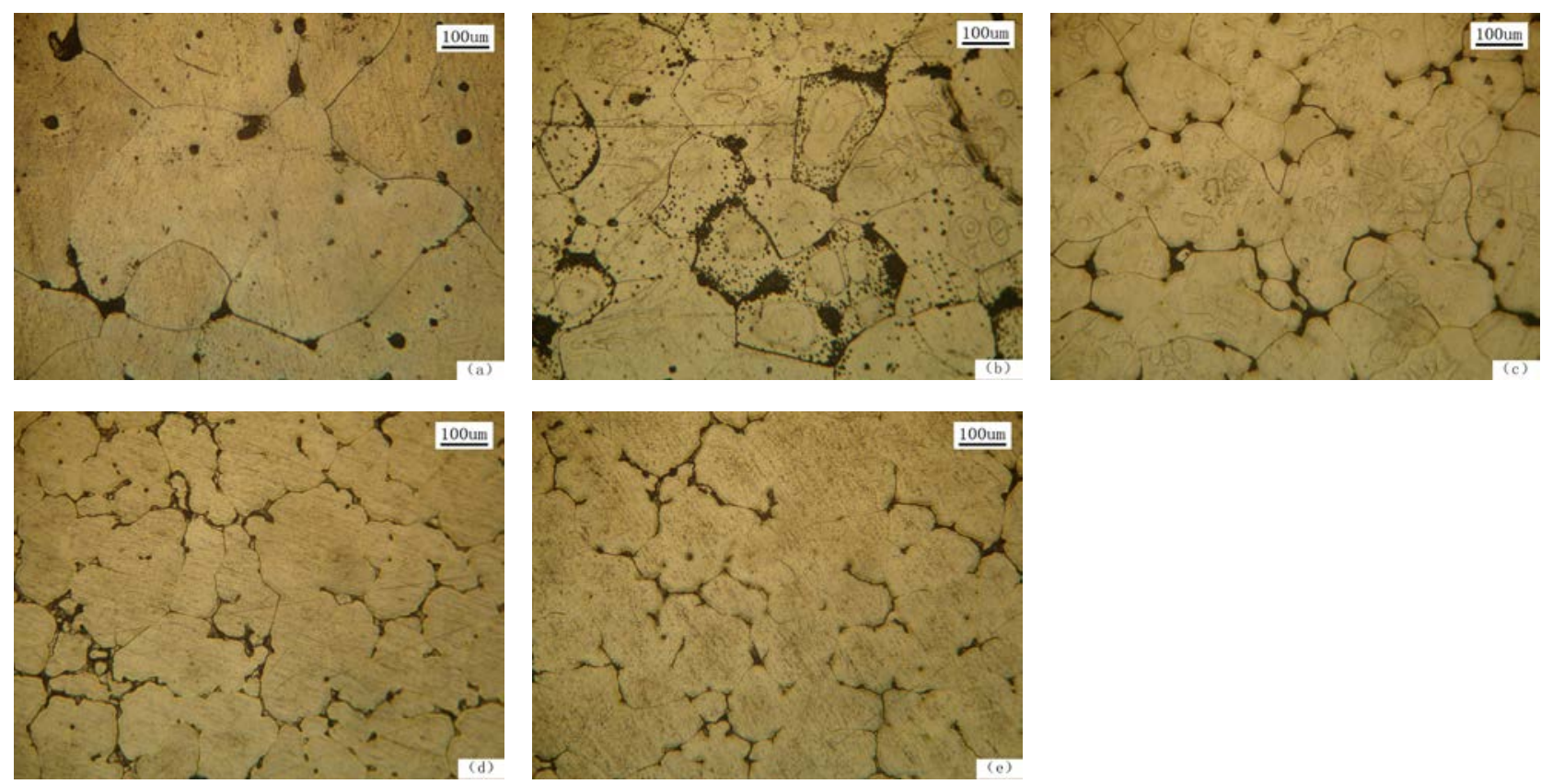

Fig.3 Micrographs of the Mg-doped aluminum ingots under UV treatment: (a) 1wt.\%, (b) 3wt.\%, (c) 5wt.\%, (d) 7wt.\% and (e) 9wt.\%, respectively.

Fig.3 shows the micrographs of Mg-doped aluminum ingots under UV treatment with the doping content is 1wt.\%, 3wt.\%, 5wt.\%, 7wt.\% and 9wt.\%, respectively. From Fig.3 we can see that with the increase of $\mathrm{Mg}$ doping content, the microstructure of grain roundness, uniform and fine, the overall trend of the average grain size with increasing alloy content is gradually reduced; when the solute content increased to 5\%, the grain size of aluminum alloy decreases sharply with increasing solute content; continue, grain size reduce speed slow. When the doped $\mathrm{Mg}$ amount is $9 \%$, the grain is most uniform and fine, we can achieve the best effect of grain refinement under ultrasonic vibration. 


\section{Conclusion}

Application of ultrasonic vibration to molten pure aluminum in solidification led to substantial grain refinement in ingot samples cooled in the air in a hot clay-graphite crucible (about $740^{\circ} \mathrm{C}$ ). Ultrasonic vibration can be used as an effective approach for grain refinement of pure aluminum. The results indicate that microstructure of the samples treated by untrosonic vibration is more finer than that of the untreated ones, with the increase of $\mathrm{Mg}$ doping, aluminum alloy molten containing 9wt.\% magnesium can achieve the best effect of grain refinement under ultrasonic vibration. This shows that solute in process of grain refinement under ultrasonic vibration conditions also played a great role. The specific mechanism of solute and ultrasonic vibration refining effects need further experimental verification.

\section{Acknowledgements}

This work has been subsidized by the Jiangxi Provincial Natural Science Foundation, China (Grant No. 20132BAB216009), the National Defense Key Discipline Laboratory of Light Alloy Processing Science and Technology foundation of China (No.gf201401006), and by the Jiangxi Provincial Educational Science Foundation, China (Grant No.GJJ14506).

\section{References}

[1] F. Wang., Z. L. Liu, D. Qiu, J. A. Taylor M. A. Easton,M. X. Zhang: Acta Mater. Vol.61 (2013), p.360.

[2] B. Chalmers, Principles of Solidification, New York: John Wiley \& Sons, 1964, 86-89.

[3] G. I. Eskin, Ultrasonic Treatment of Light Alloy Melts, London: CRC, 1998.

[4] X. Zhang, T. Inada, A. Yabe, S. Lu, Y. Kozawa: Int. J. Heat Mass Transfer Vol.44 (2001), p.4533.

[5] A. Das, M. Qian , “Grain Refinement of Magnesium Alloys by ultrasonic vibration”, Magnesium Technology 2007, (PA: TMS, 2007), 127-132.

[6] G.I. Eskin, Ultrasonic Treatment of Light Alloy Melts (Amsterdam, Gordon and Breach Science Publishers, 1998), 155.

[7] T.V. Atamanenko et al.: Metall. Mater. Trans A, Vol.41A(2010), p.2056.

[8] D.G. Eskin et al., "On the mechanism of grain refinement by ultrasonic melt treatment in the presence of transition metals,” Light metals 2010, (Warrendale, PA: TMS, 2010), 607-611.

[9] L. Zhang, D.G. Eskin, and L. Katgerman: J. Mater. Sci., Vol.46(2011), p. 5252.

[10] O.V. Abramov, Ultrasound in liquid and solid metals (Boca Raton, CRC Press, 1994), 515.

[11] L. Zhang, D.G. Eskin, A. Miroux and L. Katgerman, "Formation of microstructure in Al-Si alloys under ultrasonic melt treatment” Light metals 2012, (PA: TMS, 2012), 999-1004.

[12] X. Jian, H. Xu, T.T. Meek, and Q. Han: Scripta Mater. Vol.54(2006), p. 893. 